\title{
Optimizing the NASA Technical Report Server
}

\author{
Michael L. Nelson <m.l.nelson@larc.nasa.gov> \\ Ming-Hokng Maa <m.maa@larc.nasa.gov> \\ NASA Langley Research Center
}

\section{Abstract}

The NASA Technical Report Server (NTRS), a World Wide Web report distribution NASA technical publications service, is modified for performance enhancement, greater protocol support, and human interface optimization. Results include: Parallel database queries, significantly decreasing user access times by an average factor of 2.3; access from clients behind firewalls and/ or proxies which truncate excessively long Uniform Resource Locators (URLs); access to non- Wide Area Information Server (WAIS) databases and compatibility with the Z39-50.3 protocol; and a streamlined user interface.

\subsection{Introduction}

The original NASA Technical Report Server (NTRS) went on-line June 6, 1994 to facilitate greater distribution of NASA technical publications via the World Wide Web [Nelson, et. al., 1995]. NTRS has grown from servicing a few thousand searches a month in the beginning, to averaging over 40,000 searches per month (Figure 1). An increasing number of collections in NTRS prompted a slight user interface design (Figure 2). More importantly, the growing number of collections and increased usage required a redesign of the internal structure of NTRS to provide adequate user performance. The three initial shortcomings included:

- Slow user access times

- Non-compatibility with clients behind firewalls/proxies

- Non-compatibility with non-WAIS databases 


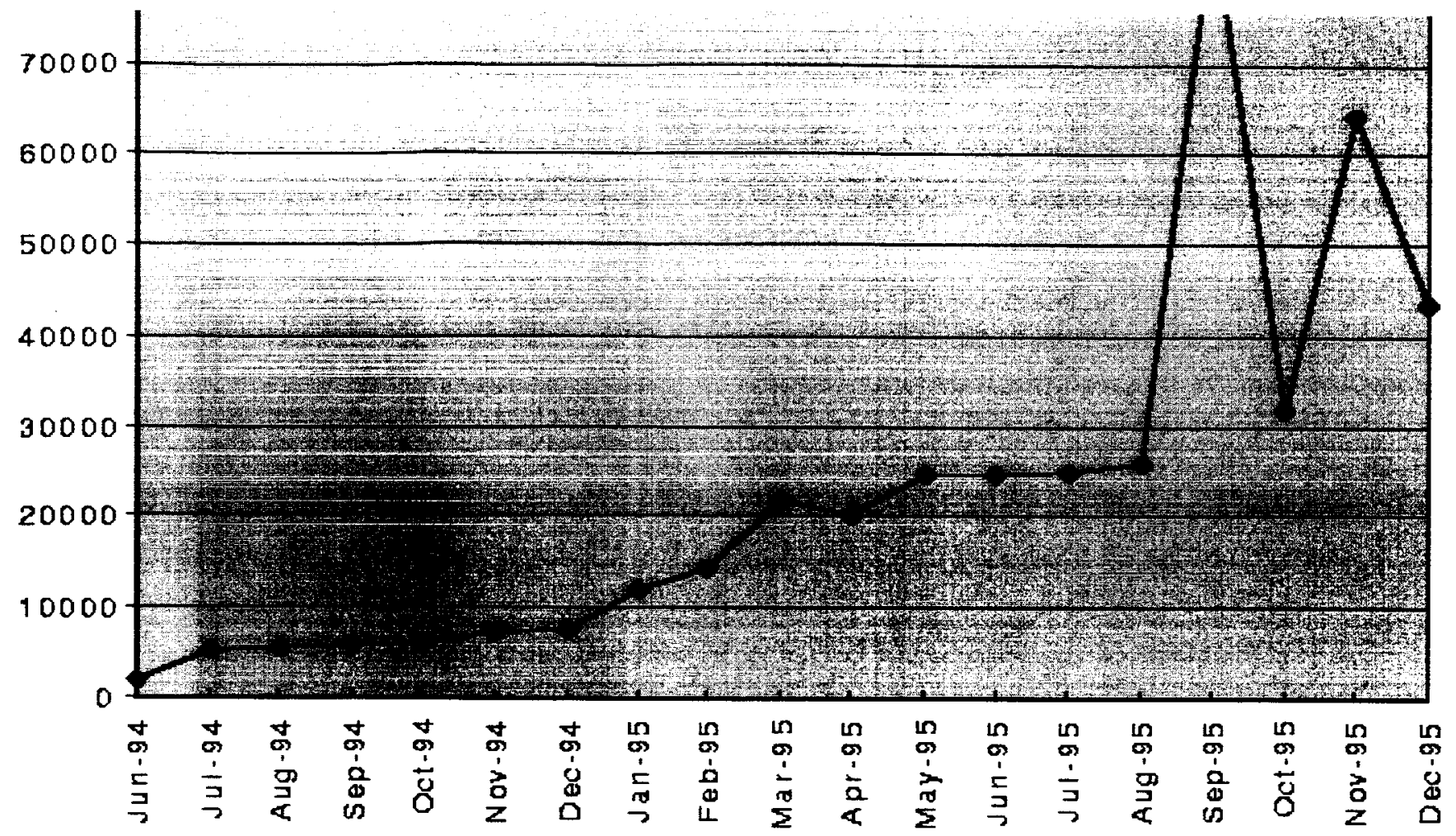

Figure 1: NTRS Accesses: June, 1994 -- Decemember 1995

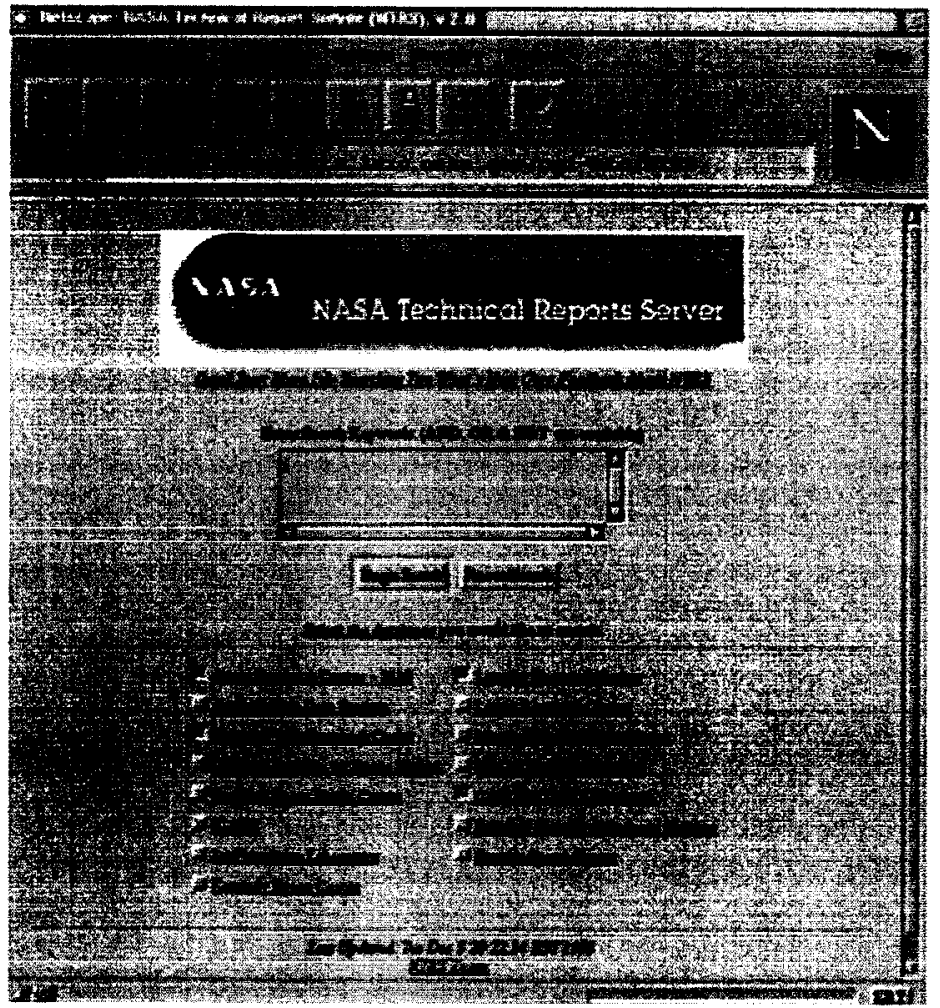

Figure 2: NTRS as seen through a WWW Browser

Because the original NTRS queried each Wide Area Information Server (WAIS) database sequentially, users of NTRS often experienced slow performance, often of 2 minutes or more for querying all available NTRS databases. Databases such as the Astrophysics Data System (ADS) and the CASI Technical Report Server (RECONselect) were especially slow due to large databases and other factors. In addition, users behind firewalls or proxies were unable to access abstracts in NTRS. Specifically, the CERN httpd proxy server canonicalizes Uniform Resource Locators (URLs), with the effect of limiting the length of URLs that can be passed through the firewall [Frystyk, 1995]. This 
results in clients attempting to access incorrect NTRS URLs that have been truncated by their proxy server. This problem is especially relevant with WAIS URLs, which can exceed 400 characters. Finally, the original NTRS was not compatible with non-WAIS query syntax. Protocols such as Z39-50.3, used in ADS, are not supported [Eichorn, et. al., 1995]. Because of these shortfalls, the original NTRS is modified to allow parallel database searches, compatibility with clients behind proxies/firewalls, and gateway compatibility with non-WAIS databases. The advantages of WAIS and non-WAIS databases are not discussed here, but can be found in [Accomazzi, Murtagh, Rasmussen 1995] and [Marchionini and Barlow. 1994]. NTRS is still available at:

http://techreports.larc.nasa.gov/cgi-bin/NTRS

\subsection{Original NTRS Architecture}

The original version of NTRS is a simple Perl script acting as a single interface to the many WAIS databases implemented by various NASA programs and centers. These databases are based on the Langley Technical Report Server [Nelson, Gottlich and Bianco, 1994]. The fundamental principle of NTRS is to have a logically central interface, but physically distributed implementation. Thus the single NTRS script provided the illusion of integrated access to various WAIS databases (Figure 2).

This implementation searches the databases sequentially. Originally, this was not a problem since the number of databases was small. But as additional NASA centers began to add their report servers, there was a need for parallel searching. Originally, few users were behind firewalls or proxies, so the URL limit went undiscovered for some time. Increased commercial usage (".com" addresses, which are often behind firewalls) exposed this problem. Finally, although ADS supports an experimental WAIS database, the bulk of their development is in Z39-50.3 (non-WAIS).

\subsection{Revised NTRS Architecture}

NTRS was revised to address the current state of: more databases, users behind firewalls, and non- WAIS databases. The NTRS architecture has lost some of its simplicity, but it now has greater flexibility (Figures 3 and 4).

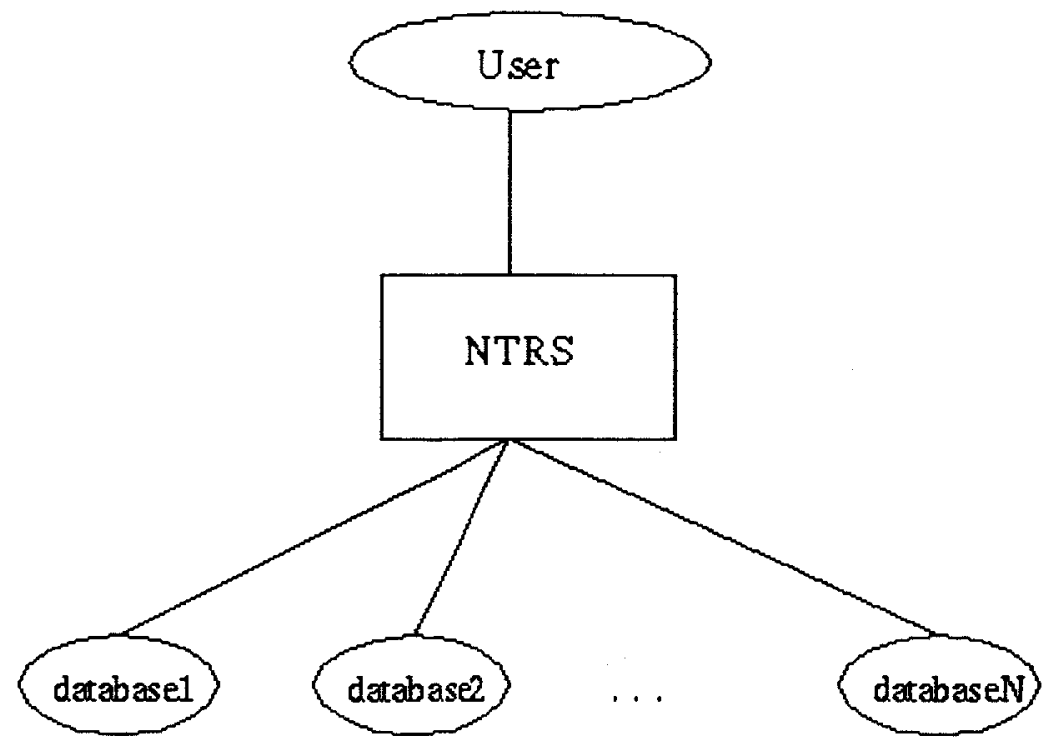

Figure 3: Architecture of Sequential NTRS 


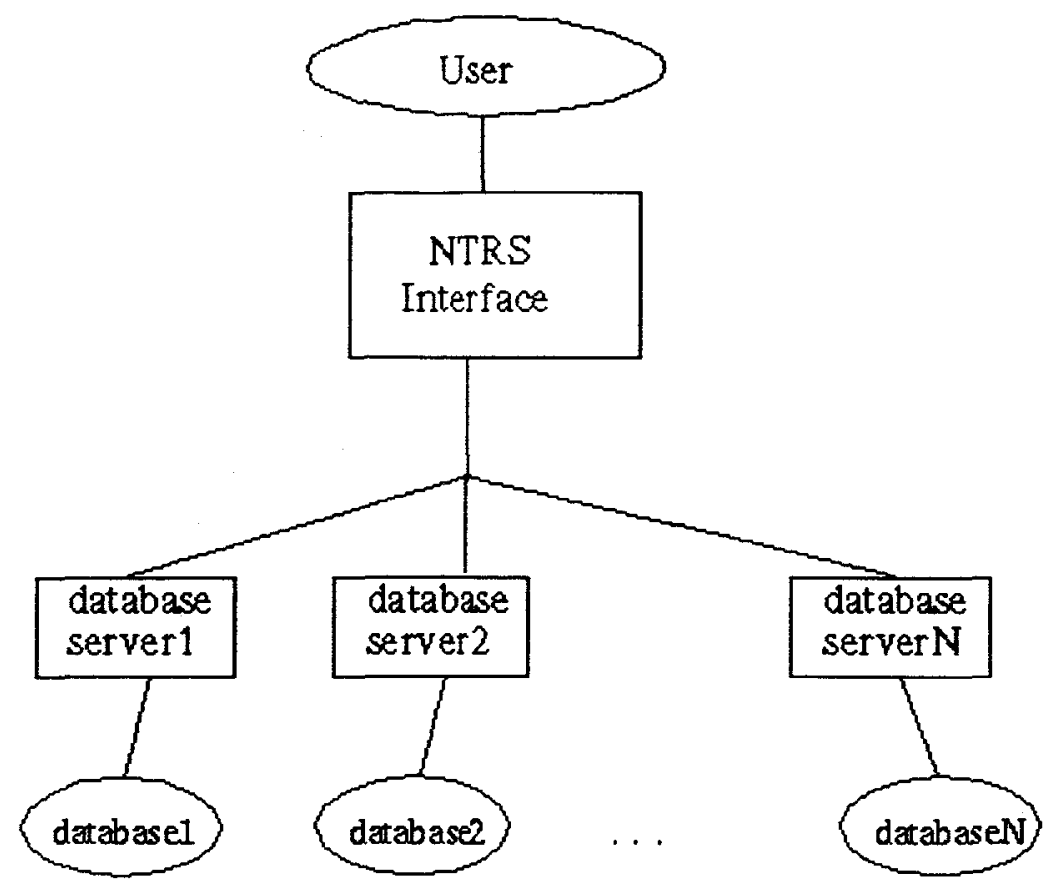

Figure 4: Architecture of Parallel NTRS

NTRS presently consists of three main parts (All written in Perl 5.000):

- User interface cgi-bin script

- 10 Database Servers, one for each database

- URL Decompression cgi-bin script

\subsection{User interface cgi-bin script}

The user interface script is the front-end to NTRS. Database queries for each database are passed sequentially by the user interface script to the appropriate database server via sockets. The user interface script then waits for the database servers to return the query results in the same order in which the queries were sent and prints the results to the user.

\subsection{Database Servers}

At first, two different methods were evaluated to implement the parallel search method: threaded and forking. Although a threaded version had greater computer science elegance, it is more complicated to implement and maintain, and thus a forking version was chosen.

Currently, CERN's httpd 3.0 has the canonicalizing feature built in, resulting in URL truncation. A patch is available to turn off the canonicalizing feature and future releases of CERN httpd will remove URL truncation. However, NTRS will keep URL compression in operation since it is not known when all copies of CERN httpd will be upgraded. Also, other firewall/proxy implementations may have similar problems.

During testing, there were 10 database servers, one for each searchable database used by NTRS. The databases servers accept client requests from the interface script. Once a request has been made, the server forks off the query to the host database and waits for the database to return the results. Once the results are collected and parsed, the server passes the query results back to the interface script for the user to view. By forking off the user queries, a query will be searched across several databases in parallel.

The server also compresses the WAIS URLs returned by the query search for users behind proxies or firewalls. Because typical WAIS URLs often contain a great deal of redundancy such as similar filepaths common to all elements in the databases, parts of the URL that are common to all documents can be replaced with a shorter token. 
On average, this compresses the size of the URLs by half, short enough to pass through proxies unharmed. The compression script also allows the retrieval to be done on the standard HTTP port (80), thus bypassing other difficulties.

Another advantage of separating the database servers into separate components is that it allows non-WAIS handling to be built in. This would have been too difficult to build into the original NTRS script.

\subsection{URL Decompression Script}

When users attempt to access a URL that has been compressed, the compressed version of the real URL is detoured first to the decompression script, which takes the compressed URL and decompresses the URL back to its original length and content. The decompression script then fetches and displays the document for the user. All of this is transparent to the user.

\subsection{Results}

Timings were taken for both the original NTRS and the new NTRS to confirm that the parallel search method produced faster results than the original sequential search method. The search words: "engine turbulence" were used. The average access time will differ depending on the number of search words queried, and the frequency of the words in the database. Ideally, if all of the NTRS databases were approximately the same speed, then the parallel method should show marked improvement. In contrast, if there were one or two slow databases while the remaining databases were relatively fast, then the parallel method would be bottlenecked by the one or two slow databases, and performance would not be as great. Tests showed that both ADS and RECON were especially slow databases, around $40+$ seconds for access (Figure 5). The rest of the databases were relatively fast, mostly under 10 seconds. Therefore, timing tests for both the sequential and parallel methods were undertaken both with and without ADS and RECON. Table 1 shows the averages for each test. In total, six tests were performed through two weeks every hour:

1. Sequential NTRS accessing all 10 databases

2. Sequential NTRS accessing all databases except ADS and RECON

3. Parallel NTRS accessing all 10 databases

4. Parallel NTRS accessing all 10 databases except ADS and RECON

5. Parallel NTRS accessing all 10 databases with proxy compression

6. Parallel NTRS accessing all 10 databases except ADS and RECON with proxy compression 


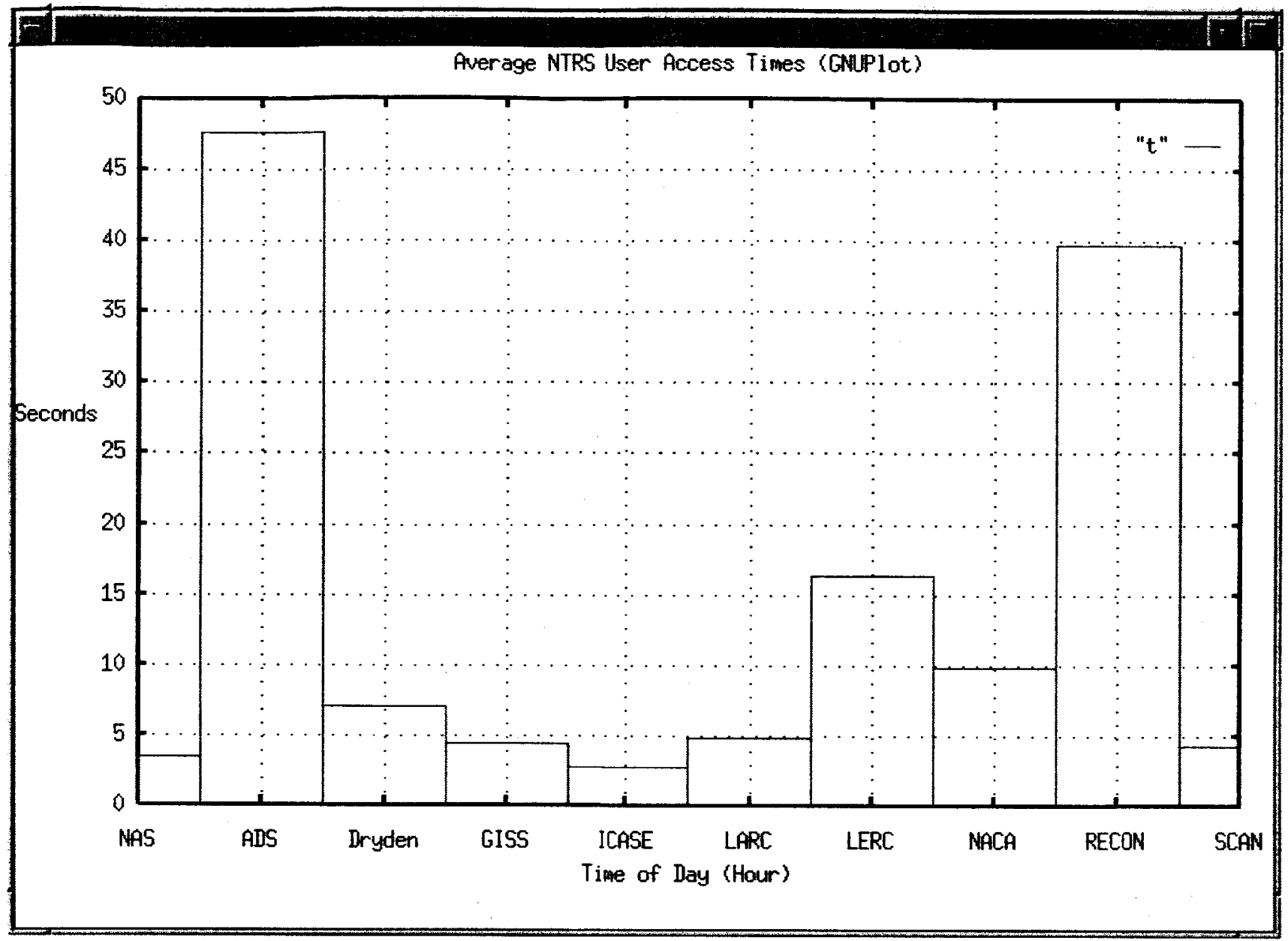

Figure 5: Access times for individual databases

\begin{tabular}{|c|c|c|}
\hline Method & All Databases & Without ADS \& RECON \\
\hline Sequential & $142.4 \mathrm{~s}$ & $52.2 \mathrm{~s}$ \\
\hline Parallel & $60.3 \mathrm{~s}$ & $22.1 \mathrm{~s}$ \\
\hline Parallel with Proxy & $87.8 \mathrm{~s}$ & $19.7 \mathrm{~s}$ \\
\hline
\end{tabular}

The results are graphed in Figures 6 - 9. The tests were run between July 17, 1995 - July 31, 1995, when the code was first implemented. The data presented does not include the optional URL compression. The tests made no attempt to compensate for network and transient conditions which would impact the timings. Not only would such compensation be difficult to model, but it would not be indicative of "real-world" performance likely to be experienced by users. Table 2 gives information about the various WAIS databases. 


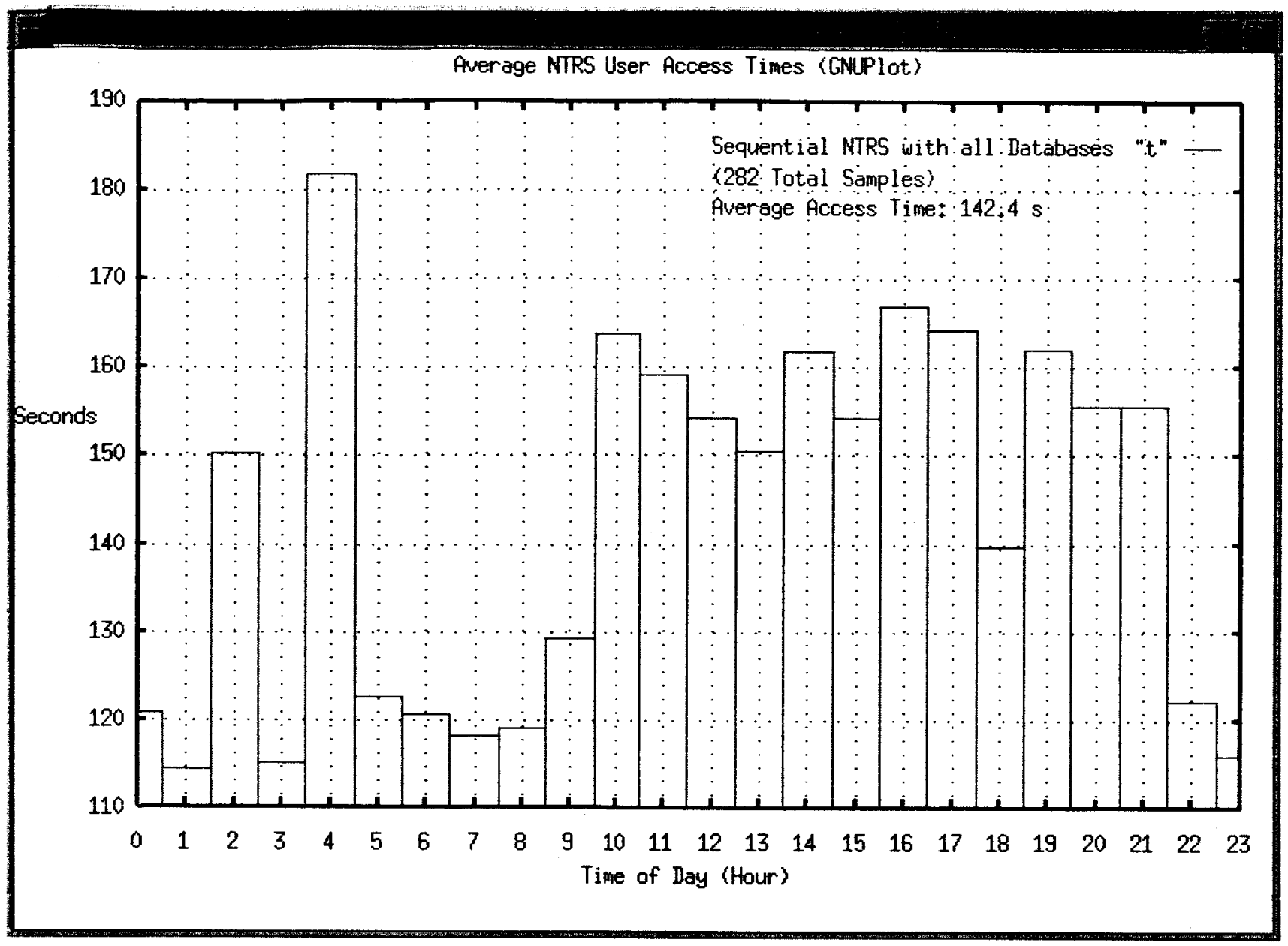

Figure 6: Access times for Sequential NTRS, all databases 


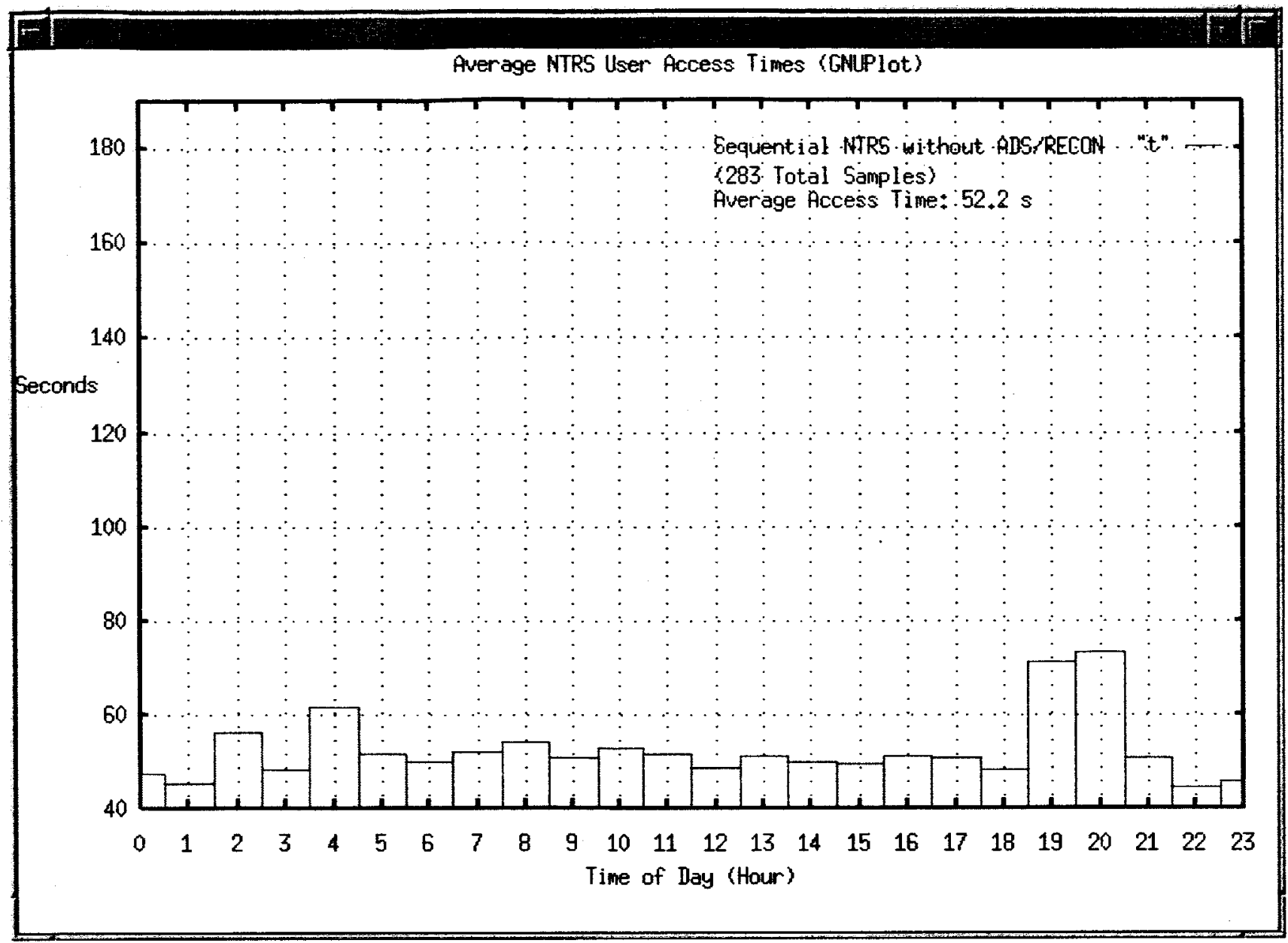

Figure 7: Access times for Sequential NTRS, all databases except ADS and RECON 


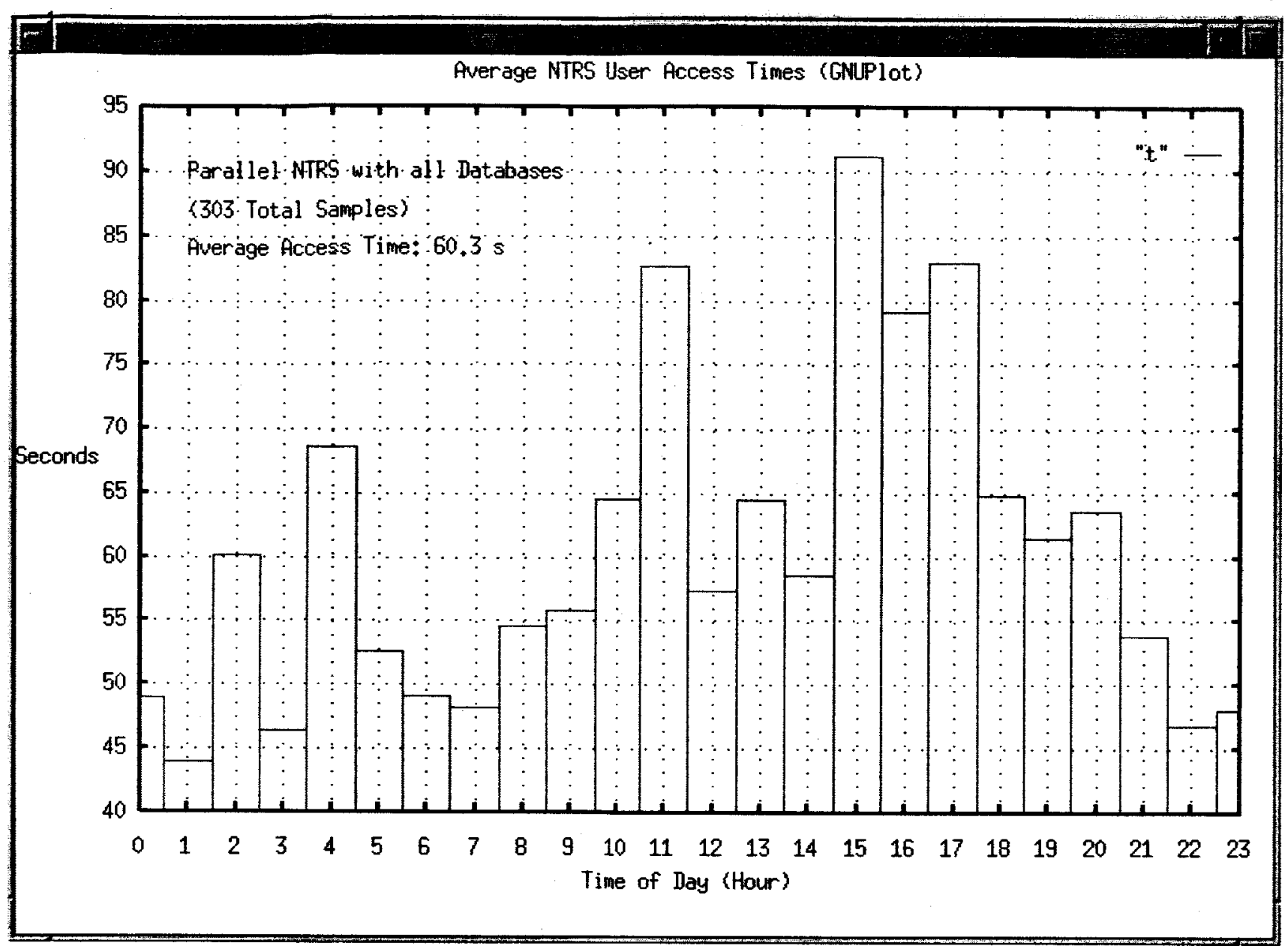

Figure 8: Access times for Parallel NTRS, all databases 


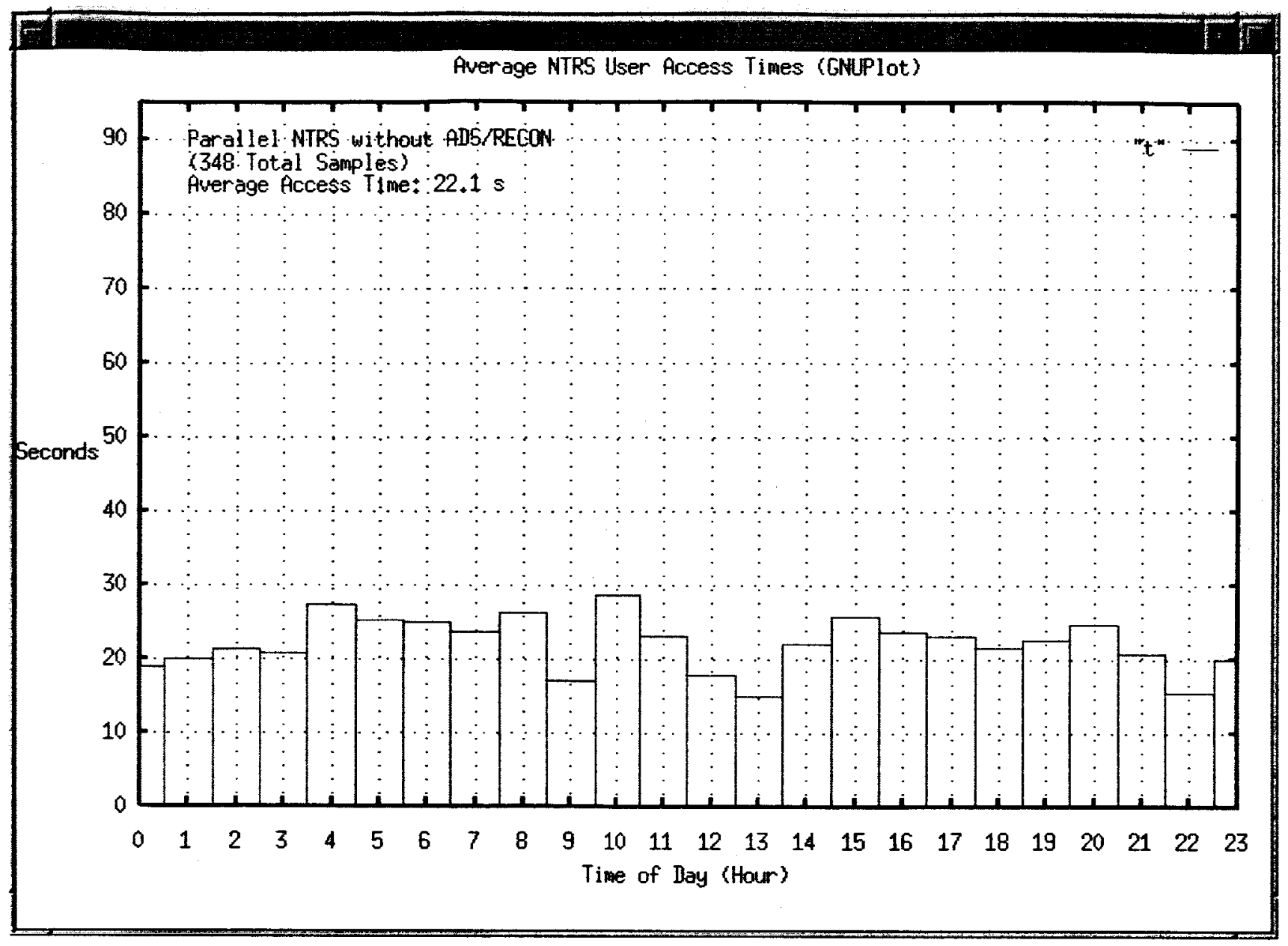

Figure 9: Access times for Parallel NTRS, all databases except ADS and RECON

\begin{tabular}{||l|l|l|l||}
\hline \multicolumn{3}{|c|}{ Table 2: Information about WAIS databases during July, 1995 } \\
\hline \hline Database & Hostname of WAIS server & Location of WAIS server & Abstracts in database \\
\hline NAS & www.larc.nasa.gov & Hampton, VA & $150+$ \\
\hline \hline ADS & adswais.harvard.edu & Cambridge, MA & $160,000+$ \\
\hline Dryden & www.dfrc.nasa.gov & Edwards, CA & $650+$ \\
\hline GISS & www.larc.nasa.gov & Hampton, VA & $550+$ \\
\hline ICASE & www.icase.edu & Hampton, VA & $150+$ \\
\hline Langley & techreports.larc.nasa.gov & Hampton, VA & $550+$ \\
\hline Lewis & letrs.lerc.nasa.gov & Cleveland, OH & $950+$ \\
\hline NACA & www.sti.nsa.gov & Linthicum Heights, MD & $13,000+$ \\
\hline RECON & www.sti.nsa.gov & Linthicum Heights, MD & $2,000,000+$ \\
\hline SCAN & www.sti.nsa.gov & Linthicum Heights, MD & $2,000+$ \\
\hline
\end{tabular}

\subsection{Discussion}

There is variation between maximum and minimum access times. This could be due to many different reasons, including:

- Timings were only collected for 2 weeks.

- The access hours for the databases unfortunately are not very well distributed. 
- Each database has its own characteristics including size of database, connection speed, host machine load, etc.

There seems to be a peak around 4 a.m. Other than that, all other high load times are during the afternoon as expected. The peak around 4 a.m. may be due to cron processes running on the server or maybe even accesses from Europe, though the actual reason for the peak remains unknown.

As expected, the differences in minimum and maximum access times for parallel NTRS without ADS and RECON is fairly small. This is due to the remaining databases having similar connection bandwidth and similar sized databases.

Parallel methods without the proxy compression have consistently improved access times by a factor of at least 2.3 for timings without ADS and RECON and for timings with all 10 databases. Perhaps the most important result is that the fastest sequential search is slower than the slowest parallel search. This holds for searches including ADS and RECON, and those excluding them.

\subsection{Future Directions}

NTRS continues to evolve. Since the time of the redesign and testing, Goddard Space Flight Center, Kennedy Space Center, Stennis Space Center, Marshall Space Flight Center and Ames Research Center have joined NTRS. In addition, the WAIS version of ADS is no longer linked from NTRS; the Z39-50.3 version of ADS has replaced it. Other Z39-50.3 databases, such as the Space Instrumentation Abstract Service, will be added to NTRS in the future.

The new database server components now allow for possible custom pre- and post-processing for each database. Small syntax differences between freeWAIS, commercial WAIS, databases with fielded searches, non-WAIS databases, etc. can now be easily hidden from the user. "Beginner" and "Expert" interfaces for NTRS are also planned, allowing NTRS to service a range of customers.

\subsection{Conclusions}

The parallel query method is faster than the original sequential query method by a factor of approximately 2.3 , or less than half the access time required. In addition, the compression method used to solve the proxying/firewall problem was implemented and user feedback indicates that it performs satisfactorily. NTRS also now includes an interface with its first non-WAIS database. The completed redesign of NTRS provides many performance enhancements and has the necessary hooks for future improvements.

Acknowledgments: Many thanks to the ICE team (http://ice-www.larc.nasa.gov/) at NASA Langley Research Center.

\section{References}

Accomazzi, A.; Murtagh, F.; Rasmussen, B. F. (1995) "Information Retrieval Tools and Techniques," Library \& Information Services in Astronomy II Conference Proceedings, European Southern Observatory, Garching, Germany, May 10-12, 1995.

Eichorn, G.; Accomazzi, A.; Grant, C. S.; Kurtz, M. J.; Murray, S. S. (1995) "Access to the Astrophysics Science Information and Abstract System," Library \& Information Services in Astronomy II Conference Proceedings, European Southern Observatory, Garching, Germany, May 10-12, 1995.

Frystyk, Henrik (1995) "Known Bugs in httpd", http://www.w3.org/hypertext/WWW/Daemon/Bugs.html.

Marchionini, G.; Barlow, D. (1994) "A Comparison of Boolean-Based Retrieval to the WAIS System for Retrieval of Aeronautical Information," NASA CR-4569.

Nelson, M. L.; Gottlich, G. L.; Bianco, D. J. (1994) "World Wide Web Implementation of the Langley Technical http://techreports.larc.nasa.gov/ltrs/papers/NASA-96-ir-p64/ 
Report Server." NASA TM-109162.

Nelson, M. L.; Gottlich, G. L.; Bianco, D. J.; Paulson, S.S.; Binkley, R. L.; Kellogg, Y. D.; Beaumont, C. J.; Schmunk, R. B.; Kurtz M. J.; and Accomazzi, A. (1995) "The NASA Technical Report Server," Internet Research: Electronic Networking Applications and Policy, vol. 5, no. 2, pp. 25-36. 